\title{
Person-Centered Trajectories of Cultural Values and Behaviors among Chinese American Adolescents
}

\author{
Moin Syed, University of Minnesota, USA \\ Linda P. Juang, University of Potsdam, Germany \\ In Press \\ Journal of Adolescence \\ Special Issue: Explaining Positive Adaptation of Immigrant Youth across Cultures
}

Address correspondence to: moin@umn.edu

We wish to acknowledge grant 1R24MH061573-01A1 from the National Institute of Health for funding to support this project, the families who participated in the study, and the research assistants who assisted with the project. 


\begin{abstract}
This study examined change in acculturation values and behavior among 310 Chinese American adolescents, and how patterns of change were related to key demographic variables and indicators of positive youth development. Dual process group-based trajectory models of change in U.S. and Chinese values and behaviors indicated a six-group solution for each. The results showed that acculturation value patterns were not related to gender, nativity, or parent education, but were related to family cohesion, self-esteem, general and academic self-efficacy, and GPA. Acculturation behavior patterns were not related to gender but were related to nativity and parent education, and were also related to general self-efficacy and family cohesion. Taken together, our findings suggest that most trajectories of development are associated with positive outcomes, but there are small groups of adolescents that function very well (those who maintain higher behavioral involvement in both) and some not very well, especially those whose behaviors are becoming more disparate over time.
\end{abstract}

Keywords: acculturation trajectories, Chinese American, positive youth development 
Acculturation research has had difficulty integrating person-centered approaches that examine configuration of processes, with longitudinal models that reveal how those processes change over time. Accordingly, much of the theoretical foundations of acculturation research have not been adequately tested. The three aims of the study address these limitations in the literature by examining acculturation trajectories among Chinese American adolescents. First, using group based trajectory modeling, we examine adolescent Chinese and U.S. acculturation trajectories in the domains of values and behaviors over time. Second, we examine the effects of

three predictors of change in acculturation, namely, gender, nativity, and parent education. Third, we test whether these trajectories are related to aspects of the self (self-esteem, general selfefficacy), academics (academic self-efficacy, GPA), and family (family cohesion). These areas represent key aspects of positive youth development, namely, confidence, competence, and connection (Lerner, 2004).

\section{Assessing Acculturation Strategies}

On an individual level, acculturation refers to the process of individual change in values, behaviors, and identities as a result of continuous contact with a new, distinct culture (Ward \& Geeaert, 2016). The bidimensional view of acculturation posits that individuals can potentially orient towards the majority culture while maintaining a strong connection to their heritage culture, and that orientation towards the majority and heritage cultures are orthogonal dimensions on which individuals vary (Berry, 2003; Schwartz et al., 2010). The nature of the variability along the two dimensions is informative of the particular acculturation strategies that individuals adopt. Much of the past literature has taken a dichotomous view of the two dimensions to create four distinct strategies: bicultural (orientation towards majority and heritage cultures), assimilated (orientation towards majority culture but not heritage), separated 
(orientation towards heritage culture but not majority), and marginalized (orientation towards neither the majority nor heritage cultures). These strategies have been studied extensively and linked to a variety of psychological and sociocultural outcomes (for review and meta-analysis see Yoon, Langrehr, \& Ong, 2011; Yoon et al., 2013).

Despite the extensive research, assessing the acculturation strategies has been a methodological challenge (Rudmin, 2003; Syed, 2013). Much of the early (and ongoing) work that focused on the four strategies used median splits of the two dimensions to categorize participants (see Ward \& Geeraert, 2016). This strategy, however, is widely understood to be statistically flawed, especially when combining two variables that have been subject to median splits (Coatsworth, Maldonado-Molina, Pantin, Szapocznik, 2005; Knight, Vargas-Chanes, Losoya, Cota-Roblles, Chassin, \& Lee, 2009). More recently, person-centered analyses, such as cluster analysis and latent profile analysis, have empirically derived acculturation profiles rather than using median splits or some other pre-specified criteria. These studies have varied in the number and nature of groups they have identified, but have generally found evidence for groups that approximate Berry's acculturation strategies (e.g., Chia \& Costigan, 2006; Choi, Tan, Yasui, \& Hahm, 2016; Weaver \& Kim, 2008). These methodological advances, however, have largely been with studies focusing on acculturation patterns at one point in time, and thus offer little to our understanding of developmental patterns.

Thinking developmentally about acculturation processes reveals a clear tension in Berry's model, as there are conceptual challenges to making sense of categories (e.g., the acculturation strategies) changing over time. Researchers have addressed this quandary by using a modelbased approach that assesses dual trajectories in acculturation processes. That is, rather than assessing how one process changes over time (e.g., change in Chinese values), dual trajectory 
models assess how two processes change in tandem (e.g., change in Chinese values and change in U.S. values). This procedure approximates the categorical nature of the acculturation strategies because the trajectory groups are defined by their relative levels and change in the two dimensions. Empirical research using this approach has generally found evidence for the different strategies, but that evidence has not been consistent, with studies finding two groups (Schwartz et al., 2015), three groups (Schwartz et al., 2013; Stoessel, Titzmann, \& Silbereisen, 2014), and four groups (Knight et al., 2014).

We return to the nature of these past results in the Discussion section, and here highlight three limitations to this past work. First, the past research examining dual acculturation trajectories has mostly included U.S. Latino samples, and has not included Asian Americans. The Asian American population, however, is the fastest growing ethnic/racial group in the U.S. (Pew Research Social and Demographic Trends, 2012), distinguished by its large percentage of young people under the age of 18 (26\%, Reeves \& Bennett, 2002). The largest Asian American group, at $23 \%$, are those of Chinese-heritage. Because of the high percentage of Chinese-heritage individuals who are born outside the U.S. (76\%, Pew Center, 2012), immigration-related issues such as acculturation are important to our understanding of Chinese American adolescent development. Indeed, Asian Americans have been at the heart of acculturation research. In a review of 138 studies of acculturation and mental health, half of the studies (51\%) focused on Asian American populations (Yoon et al., 2011). Yet, none of these studies were longitudinal. Thus, there is a need to conduct more sophisticated longitudinal models and examine withingroup variability in the acculturation experiences of Asian Americans.

The second two limitations are methodological. First, articles based on research using latent growth mixture modeling (LGMM) and group-based trajectory modeling (GBTM) tend 
not to be transparent about how different parameters are handled in the model (see Frankfurt et al., 2016). Not only are there statistical differences between LGMM and GBTM, there are conceptual ones as well. LGMM conceptualizes the identified groups as belonging to different populations of people, whereas GBTM is a method for carving up a single population into potentially meaningful subgroups (Nagin \& Odgers, 2010). Assuming that subgroups of acculturation trajectories reflect different populations, rather than variations within a population, is difficult to justify, which raises questions about the suitability of LGMM in this context. The second limitation of past work is that results of only a single model are presented in the article. There is a great deal of subjectivity when selecting the number of groups in these types of models, and oftentimes several models will fit the data equally well. The danger of this approach is that it can lead to selection of models that fit with prior conceptualization of the number and/or nature of the groups (e.g., the acculturation strategies). Thus, in the present study we not only seek to contribute to a substantive understanding of the acculturation process, but also to do so in a transparent process that addresses these past limitations.

\section{Background Predictors of Acculturation Trajectories}

By studying acculturation over time, we are also capturing adolescents' age-typical development in terms of cognitive, social, psychological, and biological changes (Michel, Titzmann, \& Silbereisen, 2012; Steinberg \& Morris, 2001). Indeed, Schönpflug (1997) has proposed that acculturation is so closely intertwined with development that to distinguish what is acculturation versus development depends on whether we are examining culture-specific processes. In addition to describing adolescent acculturation trajectories, we aim to understand predictors of acculturation trajectories. If we find predictors of the trajectories, this suggests that acculturation changes occur beyond age-typical development. The acculturation literature points 
to several important variables that might explain individual differences in acculturation trajectories, namely, adolescent gender, nativity, and parent education. Girls and boys may experience the acculturation process differently (Suárez-Orozco \& Qin, 2006). Girls, for instance, tend to maintain their heritage language (Portes \& Rumbaut, 2001) and adopt host culture values that offer greater independence and autonomy for girls more so than boys (Dion \& Dion, 2001). Adolescents' nativity, or generational status, has found to be related to acculturation trajectories. First generation Mexican-American adolescents are less likely to show a pattern of higher Mexican orientation and increasing mainstream American orientation compared to second generation (e.g., Knight et al., 2014). However, other studies have found no relation between nativity and acculturation trajectories (e.g., Updegraff et al., 2012). Parental education may also be consequential for predicting adolescent acculturation, with past studies finding that higher parent education was related to greater English competency among Russian Jewish adolescents (Birman \& Trickett, 2001). In sum, there is evidence that all of these have the potential to be predictors of acculturation trajectory groups, but because the nature of the groups was not known to us prior to the analyses, and because there is little guidance on how these background characteristics would be related to dual trajectories among Chinese Americans, we did not derive specific hypotheses.

\section{Associations with Positive Youth Development}

Acculturation stress theory has guided many of the studies linking acculturation to adjustment, postulating that the acculturation process can be difficult, and may thus lead to poorer mental health and sociocultural adaptation (e.g., Berry, 1980). In the last two decades, however, conceptualizations of acculturation emphasize the need to consider how acculturation is not always stressful, but also facilitative of more positive development in different life 
domains (Berry et al., 2006; Birman, Simon, Tran, \& Chan, 2014). For instance, greater orientation to the majority culture may facilitate greater adaptation in the school context, ultimately leading to better academic achievement (Baysu, Phalet ,\& Brown, 2011; Birman et al., 2014; Motti-Stefanidi et al., 2008; Oppedal, Røysamb, \& Sam, 2004). Adolescents from the former Soviet Union who showed greater orientation to U.S. culture also reported identifying with school more and engaging in school-related misconduct less (Trickett \& Birman, 2005). Greater heritage culture orientation may facilitate closer and more positive family relationships, thus leading to better mental health (Jasinskija-Lahti \& Liebkind, 2001; Oppedal et al., 2004; Sam \& Oppedal, 2002; Smokowski, Bacallao, \& Buchanan, 2009). Thus, orientations to majority and heritage cultures have been linked to various aspects of positive youth development in both school and family contexts.

Nonetheless, because there are still few longitudinal studies of acculturation (Knight et al., 2012, Updegraff et al., 2012), how acculturation trajectories relate to psychological and social functioning is still "open for debate" (Ward \& Geeaert, 2016). Accordingly, in the present study we examined whether trajectory groups varied across positive youth domains related to confidence, competence, and connection (Lerner, 2004): self-esteem, general self-efficacy, academic self-efficacy, GPA, and family cohesion (Fuligni, Tseng, \& Lam, 1999; Qin, 2006; Tseng, 2004).

\section{The Current Study}

We have three aims for the current study. First, we examine adolescent Chinese and U.S. acculturation trajectories in the domains of values and behaviors over time. Because acculturation values and behaviors each have different trajectories, with values being more stable compared to behaviors (Schwartz et al., 2015, van de Vijer \& Phalet, 2004), we examine both 
separately. Second, we examine whether gender, nativity, and parent education differentially predict acculturation trajectories. Third, we test whether these trajectories are related to key aspects of positive youth development.

\section{Method}

\section{Participants and Procedure}

Participants were recruited from two high schools in a large city in the U.S., each of which had a substantial number of Chinese heritage students (53\%). Potential participants were recruited from school assemblies and afterschool clubs. This yielded a total sample of 316 Chinese American adolescents (57\% female) from the two high schools who identified their father or mother to be of Chinese descent; 310 had useable data for the present study (i.e., 6 were missing demographic data). Of these, $75 \%$ completed the survey one year later (Wave 2 ) and $83 \%$ completed the survey two years later (Wave 3$)$. A cohort of ninth graders $(n=122$; mean age at Wave $1=14.2, S D=.57)$ and a cohort of tenth graders $(n=188$; mean age at Wave $1=$ $15.1, S D=.48)$ was sampled at Wave 1 . Defining our cohorts as year in school, we analyzed our data as an accelerated longitudinal design covering four time points $\left(9^{\text {th }}, 10^{\text {th }}\right.$, and $11^{\text {th }}$ grade for the first cohort; $10^{\text {th }}, 11^{\text {th }}$, and $12^{\text {th }}$ grade for the second cohort). Subsequently, we refer to four grades, which is how the data were analyzed, vs. the three waves in which the data were collected.

A majority of adolescents (64\%) were born in the U.S. and the rest outside the U.S.

Those born outside the U.S. lived in the U.S., on average, for 5.67 years $(S D=4.08)$. Most of the adolescents grew up with both parents (91\%) and most had at least one sibling (89\%).

Concerning maternal education, $4 \%$ completed elementary school or less, $11 \%$ attended middle school, 15\% attended some high school, 31\% graduated from high school, 19\% attended some 
college or university, and $20 \%$ graduated from college/university or more. Concerning paternal education, $7 \%$ completed elementary school or less, $15 \%$ attended middle school, $16 \%$ attended some high school, 28\% graduated from high school, 16\% attended some college or university, and 19\% graduated from college/university or more. Maternal and paternal education were correlated at $r=.64(p<.001)$. A new variable was created (parent education) representing the highest level of education attained by either parent. This variable was used in all analyses. More detailed descriptions of the procedure are available in a previous publication (Juang, Syed, \& Cookston 2012). The study was reviewed and approved by the ethics board of San Francisco State University.

\section{Measures}

Acculturation scale. This 50-item scale, originally designed for Vietnamese-American youth (Nguyen, Messe, \& Stollak, 1999; Nguyen \& von Eye, 2002), assesses acculturation values and behaviors along two dimensions: orientation toward majority U.S. culture (25 items; 16 values, 9 behaviors) and Chinese culture (25 items; 16 values, 9 behaviors). The scale includes a broad collection of item content, such as preferences and participation in various cultural activities, extent of contact and comfort level with American and Chinese individuals, endorsement of cultural values associated with family and gender roles, and one's overall identification with Chinese and American culture. In the current study we used two subscales, capturing values and behaviors, for each dimension (U.S. and Chinese), resulting in a total of

four subscales. The values subscales assess general endorsement of the specific item, rated on a 5-point scale from 1 (strongly disagree) to 5 (strongly agree). Sample items include, "I believe that my actions should be based primarily on the well-being of my family," and "I believe that I should do what is best for me." The behaviors subscales assess the frequency with which 
individuals engage in the behavior, rated on a 5-point scale from 1 (never) to 5 (always). Sample items include, "How often do you eat Chinese food", "How often do you watch American T.V." The four subscales (U.S. and Chinese values and behaviors) were created by calculating mean scores so that higher scores indicated greater U.S. and Chinese values and behaviors. The reliabilities for the present sample were good for the U.S. acculturation values subscale $(\alpha \mathrm{s}=$ .76-.78) and behaviors subscale $(\alpha \mathrm{s}=.80-.86)$ and Chinese acculturation values subscale $(\alpha \mathrm{s}=$ $.74-.75)$ and behaviors subscale $(\alpha \mathrm{s}=.77-.78)$.

Self-Esteem. Participants completed the 10-item Rosenberg (1989) self-esteem measure. Agreement was measured on a 4-point scale ranging from 1 (strongly disagree) to 4 (strongly agree). Negatively worded items were reverse coded and mean scores were calculated so that a higher score indicated higher self-esteem. Cronbach alphas were .84-.85.

General Self-Efficacy. General self-efficacy was measured using a 10-item scale (Schwarzer, 1993). A sample item is "I can always manage to solve problems if I try hard enough." Adolescents responded on a 4-point scale ranging from 1 (not true at all) to 4 (very true). Mean scores were calculated so that higher scores indicated greater general self-efficacy. Cronbach's alphas ranged from .85-.87.

Academic Self-Efficacy. Academic self-efficacy was measured using a 7-item scale (Bandura, Pastorelli, Barbaranelli, \& Caprara, 1999). A sample item is "How well can you get your teachers to help you when you get stuck on schoolwork?” Adolescents responded on a 5point scale ranging from 1 (not well at all) to 5 (extremely well). Mean scores were calculated so that higher scores indicated greater academic self-efficacy $(\alpha=.68)$. This measure was only assessed at Wave 3, and thus we examined differences at Grades 11 and 12 only. 
Academic performance. Adolescents' academic performance was measured using their self-reported school grade point average (GPA). Adolescents reported on a scale ranging from 0 $(F)$ to $4(A)$. A higher score represents higher academic performance.

Family cohesion. The 16-item family cohesion subscale from the Family Adaptability and Cohesion Evaluation Scales II (FACES-II; Olson, Sprenkle, \& Russell, 1979) measured the degree of connection family members have with one another. A sample item is "Family members feel very close to each other". Participants responded on a 5-point scale ranging from 1 (almost never) to 5 (almost always). A total score was computed as a sum of 16 items, with appropriate items reverse coded so that a higher score indicated a higher level of family cohesion. Cronbach's alpha for the current sample $=.80-.83$.

\section{Results}

\section{Analysis Plan}

The primary analysis consisted of two sets of dual process group-based trajectory models conducted in Mplus 7.1 (Muthén \& Muthén, 2013). One set was conducted for acculturation values and one set for acculturation behaviors. Group-based trajectory modeling (GBTM) is a longitudinal analytic technique that empirically identifies relatively homogenous subgroups of change. Because it is based on observed variables and not latent variables (as in latent growth mixture modeling), the variability within observed classes is fixed to zero. Typically, GBTM is conducted for a single variable, providing information about subgroups of change for that variable (e.g., change in U.S. values). Dual process GBTM extends the single variable case to include two variables that both change in the model (e.g., change in U.S. values and change in Chinese values at the same time). Thus, the subgroups identified through the modeling index 
similarities in the patterns of change of the two variables. That is, adolescents who change in similar ways on both variables are assigned to the same group.

Following recommended practice (Nylund, Asparouhov, \& Muthén , 2007), models were evaluated with respect to the sample size adjusted Bayesian information criterion (ssBIC), the parametric bootstrapped likelihood ratio test (BLRT), and entropy. The ssBIC is a relative indicator of model fit with lower numbers indicating better fit of the model (Raftery, 1995). The BLRT is a test based on the distribution of the log likelihood that indicates if the $k$ - 1 model should be rejected in favor of the $k$ model, (where $k$ indicates the number of classes; Nylund et al., 2007). Finally, entropy is a measure of classification accuracy, with numbers closer to 1.0 indicating perfect classification accuracy (Celeux \& Soromenho, 1993). Customary practice is to use the aforementioned criteria to arrive at the "best" fitting model. A limitation of this approach, however, is that model selection is subjective and there are often multiple models that fit the data more or less equally well. Thus, rather than using the evaluation criteria to select a single model, we used them to identify a range of plausible models. This approach helps address shortcomings of GBTM that include selection procedure, the stability of findings, and the reification of the identified groups. If conceptually coherent groups are extracted across multiple solutions, and those groups evidence the same patterns with the outcome variables, then that provides more robust evidence for the presence and significance of the group. Moreover, providing results for a range of models is a more democratic process, allowing subsequent readers to evaluate and interpret the models using their own criteria.

\section{Growth Models}

Values. Table 1 provides a summary of the evaluation criteria for models with 1-7 groups for acculturation values. The comparison of the one-group and two-group models clearly 
indicates that a group-based model is warranted. The ssBIC, entropy, and BLRT all indicate increasing utility up to the six-group solution. The ssBIC and BLRT indicated that the sevengroup solution was superior to the six-group solution, with the entropy only .01 lower, but the smallest group size was $n=2$, which is much too small. The smallest class sizes for the 4-group ( $n=13)$, 5-group $(n=11)$, and 6-group $(n=11)$ models were also small, but large enough to potentially have substantive meaning. Thus, models with 2-6 groups were retained for subsequent evaluation.

Table 2 includes intercept and slope values for the groups within the five different solutions. The identified groups were replicable across the 3- to 6-group solutions. The 2-group model was too simplistic; whereas Group 1 was identified across all five models, Group 2 separated into distinct groups in the 3-group model that persisted across all subsequent models. Thus, only the 3-6 group models were retained for further analysis.

Groups 1, 2, and 3 were evident across all four models: Group 1 consisted of adolescents relatively high on U.S. values and moderate on Chinese values; Group 2 was defined by moderate U.S. values and relatively low Chinese values; and Group 3 was defined by moderate levels of both. The 4-group model pulled out an additional, small, group with relatively low U.S. values and moderate Chinese values. The 5-group model pulled out an additional small group with U.S. values that were much higher than Chinese values, with the U.S. values increasing over time. Finally, the 6-group model pulled out another small group that was a more extreme version of the previous groups, having very high U.S. values and low Chinese values, and the Chinese values were decreasing over time. By and large, however, these groups are all marked by their stability, with very little evidence for reliable change (Figure 1). 
Behaviors. Table 1 provides a summary of the evaluation criteria for models with 1-7 groups for acculturation behaviors. While the 1-group model indicates average levels of U.S. and Chinese behaviors, the 2-group model splits this group into one with relatively higher U.S. behavior and one with relatively higher Chinese behavior, indicating the benefit of pursuing a group-based model. The ssBIC, entropy, and group sizes indicate that solutions up to six groups are plausible; the 7-group model had three group sizes less than 15. The BLRT was a less consistent indicator, suggesting that the 5-group model was not an improvement over the 4-group model, but that the 6-group model was an improvement over the 5-group model. Taken together, the data suggest the utility of further examining models with 2-6 groups.

Table 3 includes intercept and slope values for the groups within the five different solutions. The identified groups were not as stable as those in the model for acculturation values. Unique group cleavages were evident moving from two groups to three groups, but also from three groups to four groups. That is, all prior identified groups were not stable until reaching the 4-group solution. Accordingly, only the 4-6 group models were retained for further analysis.

Groups 1, 2, 3, and 4 were evident across the three solutions: Group 1 consisted of adolescents who were relatively high in both U.S. and Chinese behaviors; Group 2 had low but increasing U.S. behaviors and high Chinese behaviors; Group 3 had moderate U.S. and Chinese Behaviors; and Group 4 was defined by high U.S. behaviors relative to Chinese behaviors. Moving to the 5-group model resulted in a small group with very high levels of both behaviors. This group was also evident in the 6-group model, along with a new small group with high and increasing U.S. behaviors and low and decreasing Chinese behaviors. Although there was somewhat greater evidence for change in behaviors compared to values, the groups were still largely defined by their stability (Figure 2). 


\section{Background and Contextual Variations}

The following analyses test for whether the two sets of acculturation trajectory groups, for values and behaviors, vary by gender, immigrant generation status, and parent education. Due to number of tests the alphas level was set to .01 for all analyses.

Values. There were no differences in group membership by gender, $\chi^{2}(5)=8.89, p=.11$, $v=.17$, immigrant generation status, $\chi^{2}(5)=11.46, p=.04, v=.19$, or parent education, $F(5$, 294) $=1.96, p=.09, \eta^{2}=.03$.

Behaviors. There were no differences in group membership by gender, $\chi^{2}(5)=11.74, p=$ $.04, v=.20$, but we did observe differences for both immigrant generation status, $\chi^{2}(5)=64.55, p$ $<.001, v=.46$, and parent education, $F(5,294)=6.63, p<.001, \eta^{2}=.10$. There were many more first generation adolescents $(\mathrm{ASR}=7.5)$ in Group 2 (low increasing U.S., high Chinese), and fewer in Group 3 (moderate U.S. and Chinese; ASR = -2.9), Group 4 (high U.S., lower Chinese; ASR = -2.7), and Group 6 (high increasing U.S., low decreasing Chinese) — the latter of which included no first generation adolescents at all. The differences for parent education were more straight-forward, with Group 2 (low increasing U.S., high Chinese) coming from families with lower parent educational background compared to all other groups.

\section{Links to Positive Youth Development}

The following analyses test for whether the two sets of acculturation trajectory groups, for values and behaviors, vary in general self-efficacy, self-esteem, family cohesion, academic self-efficacy (Grades 11 and 12 only), and GPA. Due to the number of tests the alphas level was set to .01 for all omnibus tests, with Tukey's HSD tests assessing pairwise group differences.

Values. Significant differences across trajectory groups were uneven (Table 4). For general self-efficacy there were only differences at Grade 10, with Group 1 having higher levels 
than Group $2(d=0.66, p=.02)$. Self-esteem also differed only at Grade 10 , but the differences were between different groups than for general self-efficacy: Groups 2 and 4 both had lower levels than Group $6(d=-0.84, p=.01$ and $d=-1.36, p=.02$, respectively).

There were group differences in family cohesion at Grade 10 and 11. At Grade 10, Groups 1 and 3 had higher levels than Group $5(d=0.83, p=.03$ and $d=0.79, p=.006$, respectively). These same differences were observed in Grade $11(d=0.96, p=.01$ and $d=0.99$, $p=.009$, respectively), as well as Group 2 being lower than Group $3(d=0.56, p=.004)$.

There were also differences in academic self-efficacy at Grade 12 with Group 1 having higher levels than Groups 2 and $4(d=0.76, p=.001$ and $d=1.05, p<.001$, respectively). Finally, there was a significant omnibus F test for GPA at Grade 9, but the Tukey's test indicated that there were no pairwise group differences. Overall, most group differences appeared to be fleeting over time, with the exception of family cohesion, for which differences were observed over multiple grades.

Behaviors. As seen in Table 5, there were few differences among the trajectory groups. General self-efficacy differed at Grade 9, but follow up tests indicated no pairwise differences. Family cohesion differed among groups for all four grades. At both Grade 9 and 10, Group 5 was higher than Group $6(d=1.67, p=.05$ and $d=1.19, p=.05$, respectively $)$. This difference was also observed at Grade $11(d=1.62, p=.0006)$, as well as several others: Group 5 was higher than Groups $1(d=0.95, p=.04), 3(d=1.23, p=.002)$, and $4(d=1.29, p=.001)$, and Group 2 was higher than Group $6(d=0.89, p=.05)$. At Grade 12 , despite the significant omnibus test, there were no pairwise group differences. As with values, the most consistent differences were observed for family cohesion.

\section{Discussion}


The purpose of the study was to address limitations in the acculturation literature whereby static strategies of acculturation, rather than continuous development of acculturation, have been emphasized. In a new direction for the field, several studies have begun to examine acculturation trajectories over time using person-oriented analyses. The current study builds upon these recent studies by applying dual process group-based trajectory modeling to examine acculturation trajectories of Chinese American adolescents. Overall, we found evidence for a variety of different trajectory groups that, by and large, indicate stability over change. Moreover, we observed few differences by demographics and in links to positive youth development. Arguably, there are many different ways to acculturate and for most, at least within this time period, variations are not of large consequence.

\section{Two, Three, Four, Five, or Six Trajectory Groups?}

For both values and behaviors we found the strongest evidence for six trajectory groups. Although this is a greater number than what was found in previous studies (Knight et al., 2014; Schwartz et al., 2015; Stoessel et al., 2014), this was the number of groups that was suggested by the data. One possible reason for this difference is that we used group-based trajectory modeling (GBTM) and not latent growth mixture modeling (LGMM). Because GBTM relies on observed groups detected in the data, whereas LGMM identifies latent sub-groups informed by the data, GBMT tends to extract a larger number of groups compared with LGMM (Frankfurt et al., 2016; see also DiStefano \& Kamphaus, 2006).

An additional difference between our study and previous efforts is that we took a more transparent approach to modeling. Model parameters for a full range of models is provided in Tables 2 and 3; this is rarely, if ever, included in published papers, which tend to only provide the fit indices for the range of models. This transparent approach is useful for understanding how 
the conclusions of the study are related to the selected model. Importantly, the groups identified for values and behaviors were remarkably stable across models. For values there were three large groups that remained stable even into the six group solution. Groups 4-6 represented small, but potentially theoretically meaningful subgroups. The same was the case for behaviors, but four (vs. three) groups were relatively large and stable, with two very interesting small subgroups. How well do these groups map on to Berry's acculturation strategies? Overall, three of the Berry strategies appeared in our examination of trajectories that were relatively stable: integrated, assimilation, and separation over time. We did not find evidence of a stable marginalized group, which may have been indicated if mean acculturation scores were below 3 (neutral), moving into disagreeing with statements of orientation and involvement into U.S. or Chinese culture. For all groups identified, adolescents reported scores above 3 on at least one dimension, indicating some involvement in at least one or both cultures. Our findings support other reviews suggesting that a marginal group is empirically difficult to find (Ward \& Geeraert, 2016).

How well do these groups map on to past work? Some of our groups overlap with some of the groups identified in three previous studies of trajectories. In one study of Latino adolescents, latent class analysis was used to identify two groups based on acculturation behavior, values, and identity trajectories (Schwartz et al., 2015). For each dimension, there was an increasing group (increasing in both U.S. and Latino behavior, values, and identity) and a stable group (little to no change in both U.S. and Latino behavior, values, and identity). A study of Mexican American adolescents used latent class growth modeling to identify four trajectories based on acculturation values, ethnic identity, and language use: 1) high stable Mexican cultural values and moderate increasing mainstream cultural values, 2) high but decreasing Mexican 
cultural values and slight increasing mainstream cultural values, 3) high and stable Mexican cultural values and moderately high decreasing mainstream values, and 4) high and decreasing Mexican cultural with moderately high decreasing mainstream values (Knight et al., 2014). Finally, a study of German Russian adolescents used dual process growth curve modeling to identify three trajectories combining German and Russian cultural identity: 1) high and stable identification with being German and low increasing identification with being Russian, 2) low and stable identification with being German and high, decreasing identification with being Russian, and 3) moderate and stable identification with both being German and Russian (Stoessel et al., 2014). One group appears in all of these studies, including ours: moderate, stable groups in U.S. and heritage culture orientation, indicating adolescents involved in both over time. In our study, this moderate stable group was also the most common group. In contrast to stable trajectories, changes (increasing, decreasing) in each dimension and in relation to one another, are not consistent across studies. This variation indicates the great diversity of acculturation patterns that exists across adolescents. Perhaps this is not a surprising finding given the many influences that determine individual acculturation experiences (Berry et al., 2006; Ward \& Geeraert, 2016).

\section{Most Youth are Doing Just Fine: Background Predictors and Links to Positive Youth}

\section{Development}

In addition to documenting acculturation trajectories, we also focused on several factors hypothesized to predict trajectories, namely, gender, nativity, and parent education. These factors did not relate to acculturation values. For acculturation behaviors, however, nativity and parental education were related to groupings. We found that adolescents who are first generation immigrants are moving towards greater U.S. behaviors while maintaining their Chinese 
behaviors, whereas a small group of adolescents who are second generation immigrants are increasing in U.S. behaviors while moving away from Chinese behaviors. Different patterns by nativity suggest that studies of acculturation need to continue to pay attention to this key marker of diversity among immigrant adolescents. We also found that families with lower parent education were more likely to fall into a group with low increasing U.S., high stable Chinese orientations. This could be explained by the fact that first generation immigrants also came from families with less formal schooling experience. These findings highlight the importance of considering acculturation values and behaviors separately (Ward \& Geeraert, 2016).

Finally, we explored how these trajectories related to positive youth development in terms of confidence (self-esteem and general self-efficacy), competence (academic self-efficacy and GPA) and connection (family cohesion). Overall, our findings suggest that most pattern variations in acculturation trajectories do not differ greatly in their links to adjustment. For acculturation values, the pattern of associations across aspects of positive functioning did not show a clear pattern in terms of a particular group that seemed to do markedly better or worse compared to the others. For some aspects of functioning and not others, different groups did better. In one area, family cohesion, Group 5 seemed to fare the worst. This was a small group defined by diverging higher U.S. (increasing) and lower Chinese (decreasing) trajectories. For acculturation behaviors, groups did not distinguish themselves across indicators of positive functioning except for family cohesion and general self-efficacy. Group 5 (high stable on both U.S. and Chinese) seemed to fare the best in terms of higher family cohesion and self-efficacy compared to other groups, while Group 6 (high increasing U.S. and low decreasing Chinese) fared the worst. Taken together, our findings suggest that perhaps most combinations are fine, but there are small groups of adolescents that function very well (those who maintain higher 
behavioral involvement in both) and some not very well, especially those whose behaviors are becoming more disparate over time. These two groups were small, however, so conclusions should be tenuous. Adolescents who are increasingly diverging in their orientations would be in line with research on bicultural identity integration whereby individuals who have more difficulty combining both cultures (those who perhaps feel caught or conflicted between two cultures) show lower self-esteem, are less optimistic, prosocial and have poorer family relationships (Benet-Martinez \& Haritatos, 2005; Schwartz et al., 2015). Continued divergence over the years may indicate a conflicted bicultural identity that ultimately may be detrimental.

The results suggest that acculturation change is a slow process, at least for the three year time period we assessed. We also may have seen greater acculturative change among adolescents along the U.S. dimension if we had sampled from a community that had, for instance, a high European American population and few Chinese Americans. The families in our study lived in a very strong Chinese community consisting of a large and vibrant Chinatown and various institutions (e.g., language schools, churches, media, restaurants, community centers) geared towards the Chinese population. Further, the adolescents attended schools that had an even higher concentration of Chinese and Asian peers than in the broader community. Our results suggest that in a strong, ethnically cohesive city and school context, immigrant adolescents show orientations to both cultures simultaneously and that various combinations of orientations over time did not lead to markedly different adjustment patterns. Continued attention to the broader community context to understand the nature and extent of acculturation to the heritage and majority culture is needed (Berry et al., 2006).

Alternatively, perhaps our measure of acculturation values and behaviors masked more nuanced changes in U.S. acculturation over time. For instance, one study found that only some 
aspects of acculturation changed over a one-year period for Russian Jewish adolescents in the U.S. More specifically, English language competence changed (improved), but American behavioral acculturation and identity did not (Birman \& Trickett, 2001). At this time it is not fully known what the appropriate conceptual domains are, nor how to assess them, but it would be useful for future research to examine specific areas of acculturation separately (see also Zane \& Mak, 2003), and in different community contexts, to assess whether some areas may be more susceptible to change than others (see also Berry \& Sabatier, 2011, and Rudmin, 2009, for excellent discussions of measurement issues in acculturation research).

\section{Limitations and Future Research}

Several limitations to the study should be noted. First, only survey data were used. Indepth interviews would have offered another, richer portrait of the lives of Chinese American adolescents to uncover the complexities of acculturation. Doing so will offer more insight into the actual processes that may explain the pattern of acculturation trajectories. Second, the time span of our study was limited. Longer studies across transitions would allow for more opportunities to identify fluctuations in patterns of acculturation. Nonetheless, because there are still so few longitudinal studies of individual patterns of acculturation, our study adds to the literature by describing patterns for Chinese American adolescents. Third, some of the trajectory groups identified were small. Due to small groups, we could really only detect large effects between groups. However, in many ways, large effects are perhaps all we should really care about. Small effects may be meaningful differences, and may have implications, but they could also be consistent with the notion that just about any trajectory is mostly adaptive. Thus, we observed variations in normative patterns of development. This can be seen in the mean levels for all the groups on variables - none are especially low. Another limitation associated with the 
small groups, however, is that they were quite variable, with relatively large standard deviations. This raises the question of how internally consistent such groups is, a valid criticism for all analyses that seek to classify continuous processes. Fourth, this study was conducted in a diverse urban setting with a long history of Chinese immigrants and a high density of Chinese.

Consequently, this context provides more cultural support and opportunities for adolescents to maintain their Chinese culture than locations with fewer Chinese populations. Perhaps in other community contexts we would find different groups, perhaps we would see patterns defined by a more rapid decrease in orientation toward Chinese culture and/or more rapid rate of increase in orientation toward U.S. culture. Future research should draw samples from communities that vary by cultural support to test how contexts modify the acculturation process.

\section{Conclusion}

Acculturation change is a slow process; it is also quite variable. Chinese American adolescents showed a variety of patterns of acculturation with the most common pattern being of stable, moderate involvement in both U.S. and Chinese cultures over time. Importantly, our indicators of confidence, competence, and connection (self, school, and family adjustment) were not tied closely to the different groupings, suggesting that there are many different ways in which adolescents can incorporate U.S. and Chinese cultures in their lives and that there is perhaps no single optimal acculturation trajectory. There may, however, be one less optimal pattern, the small group of adolescents showing increasingly diverging trajectories. Overall, however, our data show that various acculturation trajectories are mostly adaptive. 


\section{References}

Bandura, A., Pastorelli, C., Barbaranelli, C., \& Caprara, G. V. (1999). Self-efficacy pathways to childhood depression. Journal of Personality and Social Psychology, 76(2), 258-269.

Benet-Martínez, V., \& Haritatos, J. (2005). Bicultural identity integration (BII): Components and psychosocial antecedents. Journal of Personality, 73(4), 1015-1050.

Berry, J.W. (1980). Acculturation as varieties of adaptation. In A.M. Padilla (Ed.), Acculturation: Theory, models and some new findings, (pp. 9-25). Boulder: Westview.

Berry, J. W. (2003). Conceptual approaches to acculturation. In K. Chun, P. Balls-Organista, \& G. Martin (Eds.), Acculturation: Advances in theory, measurement, and applied research (pp. 17-37). Washington: American Psychological Association Press.

Berry, J. W., Phinney, J. S., Sam, D. L., \& Vedder, P. (Eds.). (2006). Immigrant youth in cultural transition: Acculturation, identity, and adaptation across national contexts. Mahwah, NJ: Lawrence Erlbaum Associates Publishers.

Berry, J. W., \& Sabatier, C. (2011). Variations in the assessment of acculturation attitudes: Their relationships with psychological wellbeing. International Journal of Intercultural Relations, 35(5), 658-669.

Baysu, G., Phalet, K., \& Brown, R. (2011). Dual identity as a two-edged sword: Identity threat and minority school performance. Social Psychology Quarterly, 74(2), 121-143. doi:10.1177/0190272511407619

Birman, D., Simon, C. D., Chan, W. Y., \& Tran, N. (2014). A life domains perspective on acculturation and psychological adjustment: A study of refugees from the former Soviet Union. American Journal of Community Psychology, 53(1-2), 60-72. 
Birman, D., \& Trickett, E. J. (2001). Cultural transitions in first-generation immigrants acculturation of Soviet Jewish refugee adolescents and parents. Journal of Cross-Cultural Psychology, 32(4), 456-477.

Celeux, G., \& Soromenho, G. (1996). An entropy criterion for assessing the number of clusters in a mixture model. Journal of Classification, 13(2), 195-212.

Chia, A.-L., \& Costigan, C. L. (2006). A person-centred approach to identifying acculturation groups among Chinese Canadians. International Journal of Psychology, 41(5), 397-412.

Choi, Y., Tan, K. P. H., Yasui, M., \& Hahm, H. C. (2016). Advancing understanding of acculturation for adolescents of Asian immigrants: Person-oriented analysis of acculturation strategy among Korean American youth. Journal of Youth and Adolescence, $45(7), 1380-1395$.

Coatsworth, J. D., Maldonado-Molina, M., Pantin, H., \& Szapocznik, J. (2005). A PersonCentered and Ecological Investigation of Acculturation Strategies in Hispanic Immigrant Youth. Journal of Community Psychology, 33(2), 157-174.

Costigan, C., \& Dokis, D. (2006). Relations between parent-child acculturation differences and adjustment within immigrant Chinese families. Child Development, 77(5), 1252-1267.

Dion, K. K., \& Dion, K. L. (2001). Gender and cultural adaptation in immigrant families. Journal of Social Issues, 57(3), 511-521.

DiStefano, C., \& Kamphaus, R. W. (2006). Investigating subtypes of child development a comparison of cluster analysis and latent class cluster analysis in typology creation. Educational and Psychological Measurement, 66(5), 778-794. 
Frankfurt, S., Frazier, P. F., Syed, M., \& Jung, K. R. (2016). Using group-based trajectory and growth mixture modeling to identity change trajectory classes. The Counseling Psychologist, 44(5), 622-660.

Fuligni, A. J., Tseng, V., \& Lam, M. (1999). Attitudes toward family obligations among American adolescents with Asian, Latin American, and European backgrounds. Child Development, 70(4), 1030-1044.

Jasinskaja-Lahti, I., \& Liebkind, K. (2001). Perceived discrimination and psychological adjustment among Russian-speaking immigrant adolescents in Finland. International Journal Of Psychology, 36(3), 174-185. doi:10.1080/00207590042000074

Juang, L. P., Syed, M., \& Cookston, J. T. (2012). Acculturation-based and everyday parentadolescent conflict among Chinese American adolescents: Longitudinal trajectories and implications for mental health. Journal of Family Psychology, 26(6), 916-926.

Kim, S. Y., Chen, Q., Wang, Y., Shen, Y., \& Orozco-Lapray, D. (2013). Longitudinal linkages among parent-child acculturation discrepancy, parenting, parent-child sense of alienation, and adolescent adjustment in Chinese immigrant families. Developmental Psychology, 49(5), 900-912.

Knight, G. P., Vargas-Chanes, D., Losoya, S. H., Cota-Robles, S., Chassin, L., \& Lee, J. M. (2009). Acculturation and enculturation trajectories among Mexican-American adolescent offenders. Journal of Research on Adolescence, 19(4), 625-653.

Knight, G. P., Basilio, C. D., Cham, H., Gonzales, N. A., Liu, Y., \& Umaña-Taylor, A. J. (2014). Trajectories of Mexican American and mainstream cultural values among Mexican American adolescents. Journal of Youth and Adolescence, 43(12), 2012-2027. 
Michel, A., Titzmann, P. F., \& Silbereisen, R. K. (2012). Psychological adaptation of adolescent immigrants from the former Soviet Union in Germany: Acculturation versus age-related time trends. Journal Of Cross-Cultural Psychology, 43(1), 59-76. doi:10.1177/0022022111416662

Muthén, L. K., \& Muthén, B. O. (2013). Mplus 7.11. Los Angeles, CA: Muthén \& Muthén.

Nagin, D. S., \& Odgers, C. L. (2010). Group-based trajectory modeling in clinical research. Annual Review of Clinical Psychology, 6, 109-138.

Nguyen, A.-M. D., \& Benet-Martínez, V. (2013). Biculturalism and adjustment: A metaanalysis. Journal of Cross-Cultural Psychology, 44(1), 122-159.

Nguyen, H. H., Messé, L. A., \& Stollak, G. E. (1999). Toward a more complex understanding of acculturation and adjustment. Journal of Cross-Cultural Psychology, 30(1), 5-31.

Nguyen, H. H., \& von Eye, A. (2002). The Acculturation Scale for Vietnamese Adolescents (ASVA): A bidimensional perspective. International Journal Of Behavioral Development, 26(3), 202-213. doi:10.1080/01650250042000672

Nylund, K. L., Asparouhov, T., \& Muthén, B. O. (2007). Deciding on the number of classes in latent class analysis and growth mixture modeling: A Monte Carlo simulation study. Structural Equation Modeling, 14(4), 535-569.

Olson, D.H., Sprenkle; D.H. \& Russell, C.S. (1979). Circumplex model of marital and family systems: Cohesion and adaptability dimensions, family types, and clinical applications. Family Process, 18, 3-28.

Oppedal, B., Røysamb, E., \& Sam, D. L. (2004). The effect of acculturation and social support on change in mental health among young immigrants. International Journal Of Behavioral Development, 28(6), 481-494. doi:10.1080/01650250444000126 
Pew Research Social and Demographic Trends. (2012). The rise of Asian Americans. Retrieved from http:/www.pewsocialtrends.org/2012/06/19/the-rise-of-asian-americans/

Phinney, J.S., Berry, J. W., Vedder, P., \& Liebkind, K. (2006). The acculturation experience: Attitudes, identities, and behaviors of immigrant youth. In J. W. Berry, J. S. Phinney, D. Sam, D., \& P. Vedder (Eds.), Immigrant youth in cultural transition: Acculturation, identity and adaptation across national contexts, pp. 71-116. New Jersey: Lawrence Erlbaum.

Portes, A., Fernández-Kelly, P., \& Haller, W. (2005). Segmented assimilation on the ground: The new second generation in early adulthood. Ethnic and Racial Studies, 28(6), 10001040.

Portes, A. \& Rumbaut, R. (2001). Legacies: The story of the second generation. Berkeley: University of California Press.

Qin, D. (2006). Our child doesn't talk to us anymore': Alienation in immigrant Chinese families. Anthropology \& Education Quarterly, 37(2), 162-179.

Raftery, A. E. (1995). Bayesian model selection in social research. Sociological Methodology, 111-163.

Reeves, T., \& Bennett, C. (2002). The Asian and Pacific Islander Population in the United States: March 2002. Population, 20(540), 5.

Rosenberg, M. (1989). Society and the adolescent self-image. Middletown, CT: Wesleyan University Press (Revised edition).

Rudmin, F. W. (2003). Critical history of the acculturation psychology of assimilation, separation, integration, and marginalization. Review of General Psychology, 7(1), 3. 
Rudmin, F. (2009). Constructs, measurements and models of acculturation and acculturative stress. International Journal of Intercultural Relations, 33(2), 106-123.

Schönpflug, U. (1997). Acculturation: Adaptation or development?. Applied Psychology: An International Review, 46(1), 52-55. doi:10.1080/026999497378511

Schwartz, S. J., Des Rosiers, S., Huang, S., Zamboanga, B. L., Unger, J. B., Knight, G. P., ... \& Szapocznik, J. (2013). Developmental trajectories of acculturation in Hispanic adolescents: Associations with family functioning and adolescent risk behavior. Child Development, 84(4), 1355-1372.

Schwartz, S. J., Unger, J. B., Zamboanga, B. L., Córdova, D., Mason, C. A., Huang, S., . . Szapocznik, J. (2015). Developmental trajectories of acculturation: Links with family functioning and mental health in recent immigrant Hispanic adolescents. Child Development, 86(3), 726-748.

Schwartz, S. J., Unger, J. B., Zamboanga, B. L., \& Szapocznik, J. (2010). Rethinking the concept of acculturation: Implications for theory and research. American Psychologist, 65(4), $237-251$

Schwarzer, R. (1993). Measurement of perceived self-efficacy. Psychometric scales for crosscultural research. Berlin, Germany: Freie Universität Berlin.

Smokowski, P. R., Bacallao, M., \& Buchanan, R. L. (2009). Interpersonal mediators linking acculturation stressors to subsequent internalizing symptoms and self-esteem in Latino adolescents. Journal Of Community Psychology, 37(8), 1024-1045. doi:10.1002/jcop.20346

Steinberg, L., \& Morris, A. S. (2001). Adolescent development. Annual Review Of Psychology, 5283-110. doi:10.1146/annurev.psych.52.1.83 
Stoessel, K., Titzmann, P. F., \& Silbereisen, R. K. (2014). Being 'them' and 'us' at the same time? Subgroups of cultural identification change among adolescent diaspora immigrants. Journal of Cross-Cultural Psychology, 45(7), 1089-1109.

Stonequist, E.V. (1937). The marginal man: A study in personality and culture conflict. New York: Russell \& Russell.

Suárez-Orozco, C. \& Qin, D. (2006). Gendered perspectives in psychology: Immigrant origin youth. International Migration Review, 40(1), 165-198.

Syed, M. (2013). Assessment of ethnic identity and acculturation. In K. Geisinger (Ed.), APA handbook of testing and assessment in psychology (pp. 393-405). Washington DC: American Psychological Association.

Trickett, E. J., \& Birman, D. (2005). Acculturation, School Context, and School Outcomes: Adaptation of Refugee Adolescents From the Former Soviet Union. Psychology In The Schools, 42(1), 27-38. doi:10.1002/pits.20024

Tseng, V. (2004). Family interdependence and academic adjustment in college: Youth from immigrant and U.S.-born families. Child Development, 75(3), 966-983.

Updegraff, K. A., Umaña Taylor, A. J., McHale, S. M., Wheeler, L. A., \& Perez Brena, N. J. (2012). Mexican-origin youth's cultural orientations and adjustment: Changes from early to late adolescence. Child Development, 83(5), 1655-1671.

van de Vijver, F. J. R., \& Phalet, K. (2004). Assessment in multicultural groups: The role of acculturation. Applied Psychology: An International Review, 53(2), 215-236.

Ward, C., \& Geeraert, N. (2016). Advancing acculturation theory and research: the acculturation process in its ecological context. Current Opinion in Psychology, 8, 98-104. 
Weaver, S. R., \& Kim, S. Y. (2008). A person-centered approach to studying the linkages among parent-child differences in cultural orientation, supportive parenting, and adolescent depressive symptoms in Chinese American families. Journal of Youth and Adolescence, $37(1), 36-49$.

Yeh, C. J. (2003). Age, acculturation, cultural adjustment, and mental health symptoms of Chinese, Korean, and Japanese immigrant youths. Cultural Diversity \& Ethnic Minority Psychology, 9(1), 34-48.

Yoon, E., Chang, C.-T., Kim, S., Clawson, A., Cleary, S. E., Hansen, M., . . Gomes, A. M. (2013). A meta-analysis of acculturation/enculturation and mental health. Journal of Counseling Psychology, 60(1), 15-30.

Yoon, E., Langrehr, K., \& Ong, L. Z. (2011). Content analysis of acculturation research in counseling and counseling psychology: A 22-year review. Journal of Counseling Psychology, 58(1), 83-96.

Zane, N \& Mak, W. (2003). Major approaches to the measurement of acculturation among ethnic minority populations: A content analysis and an alternative empirical strategy. In K. Chun, P. Organista, \& G. Marin (Eds.), Acculturation: Advances in Theory, Measurement and Applied Research (pp. 39-60). Washington, DC: American Psychological Association. 
Table 1

Model Fit Indices for GBTM Analyses

\begin{tabular}{lcccccc}
\hline Values & AIC & BIC & ssBIC & Entropy & BLRT & $\begin{array}{c}\text { Smallest } \\
\text { Group }\end{array}$ \\
\hline 1 group & 1703.49 & 1748.33 & 1710.27 & 1.00 & & 310 \\
2 group & 1552.21 & 1615.73 & 1561.82 & 0.64 & $161.280(.0000)$ & 97 \\
3 group & 1487.50 & 1569.70 & 1499.93 & 0.67 & $74.713(.0000)$ & 42 \\
4 group & 1421.10 & 1521.99 & 1436.36 & 0.74 & $76.395(.0000)$ & 13 \\
5 group & 1363.99 & 1483.56 & 1382.07 & 0.77 & $67.118(.0000)$ & 11 \\
6 group & 1351.73 & 1489.98 & 1372.63 & 0.78 & $22.261(.0000)$ & 11 \\
7 group & 1336.53 & 1493.46 & 1360.25 & 0.77 & $25.241(.0128)$ & 2 \\
\hline Behaviors & AIC & BIC & ssBIC & Entropy & BLRT $(p)$ & Smallest \\
\hline 1 group & 3103.58 & 3148.42 & 3110.36 & 1.00 & & 310 \\
\hline group & 2776.59 & 2840.11 & 2786.19 & 0.78 & $336.990(.0000)$ & 123 \\
3 group & 2659.54 & 2741.75 & 2671.97 & 0.81 & $127.048(.0144)$ & 62 \\
4 group & 2510.27 & 2611.16 & 2525.52 & 0.81 & $159.272(.0027)$ & 58 \\
\hline group & 2469.85 & 2589.42 & 2487.92 & 0.80 & $50.423(.1959)$ & 16 \\
6 group & 2450.06 & 2588.31 & 2470.96 & 0.80 & $29.788(.0000)$ & 13 \\
\hline group & 2427.39 & 2584.32 & 2451.12 & 0.78 & $32.669(.0000)$ & 12 \\
\hline
\end{tabular}


Table 2

Growth Parameters for Values Groups across Models

\begin{tabular}{|c|c|c|c|c|c|c|c|c|c|c|c|c|c|c|c|c|c|c|c|}
\hline \multirow[b]{2}{*}{ Model } & & \multicolumn{3}{|c|}{ Group 1} & \multicolumn{3}{|c|}{ Group 2} & \multicolumn{3}{|c|}{ Group 3} & \multicolumn{3}{|c|}{ Group 4} & \multicolumn{3}{|c|}{ Group 5} & \multicolumn{3}{|c|}{ Group 6} \\
\hline & & $n$ & 1 & $S$ & $n$ & 1 & $S$ & $n$ & I & $S$ & $n$ & I & $S$ & $n$ & 1 & $S$ & $n$ & 1 & $S$ \\
\hline \multirow[t]{2}{*}{1} & U.S. & $\begin{array}{c}31 \\
0\end{array}$ & 3.638 & $\begin{array}{c}- \\
0.018\end{array}$ & & & & & & & & & & & & & & & \\
\hline & Ch. & & 3.393 & $\begin{array}{c}- \\
0.015\end{array}$ & & & & & & & & & & & & & & & \\
\hline \multirow[t]{2}{*}{2} & U.S. & $\begin{array}{c}21 \\
3\end{array}$ & 3.467 & $\begin{array}{c}- \\
0.019\end{array}$ & 97 & 3.895 & 0.029 & & & & & & & & & & & & \\
\hline & Ch. & & 3.49 & $\begin{array}{c}- \\
0.016\end{array}$ & & 3.245 & -0.037 & & & & & & & & & & & & \\
\hline \multirow[t]{2}{*}{3} & U.S. & 42 & 4.087 & 0.056 & 107 & 3.681 & -0.001 & 161 & 3.427 & -0.014 & & & & & & & & & \\
\hline & Ch. & & 3.428 & 0.027 & & 3.154 & -0.029 & & 3.593 & -0.027 & & & & & & & & & \\
\hline \multirow[t]{2}{*}{4} & U.S. & 40 & 4.106 & 0.057 & 119 & 3.654 & 0.006 & 138 & 3.504 & -0.029 & 13 & 2.671 & 0.103 & & & & & & \\
\hline & Ch. & & 3.389 & $\begin{array}{c}- \\
0.017\end{array}$ & & 3.172 & -0.031 & & 3.645 & -0.026 & & 3.332 & -0.073 & & & & & & \\
\hline \multirow[t]{2}{*}{5} & U.S. & 36 & 4.077 & 0.043 & 158 & 3.587 & -0.022 & 75 & 3.459 & -0.014 & 11 & 2.521 & 0.12 & 30 & 3.851 & $0.089 *$ & & & \\
\hline & Ch. & & 3.461 & 0.011 & & 3.323 & -0.036 & & 3.751 & -0.017 & & 3.336 & -0.068 & & 2.951 & -0.067 & & & \\
\hline \multirow[t]{2}{*}{6} & U.S. & 30 & 3.952 & 0.088 & 160 & 3.597 & -0.026 & 71 & 3.424 & -0.003 & 11 & 2.53 & 0.121 & 20 & 3.721 & 0.081 & 18 & 4.299 & -0.006 \\
\hline & Ch. & & 3.553 & 0.018 & & 3.336 & -0.039 & & 3.742 & -0.009 & & 3.33 & -0.066 & & 2.961 & $-0.107^{*}$ & & 3.157 & -0.033 \\
\hline
\end{tabular}

Note. Continuously shaded and continuously white columns indicate stable groups across solutions (i.e., groups remained stable as of Model 3). 
Table 3

Growth Parameters for Behaviors Groups across Models

\begin{tabular}{|c|c|c|c|c|c|c|c|c|c|c|c|c|c|c|c|c|c|c|c|}
\hline \multirow[b]{2}{*}{ Model } & & \multicolumn{3}{|c|}{ Group 3} & \multicolumn{3}{|c|}{ Group 1} & \multicolumn{3}{|c|}{ Group 2} & \multicolumn{3}{|c|}{ Group 4} & \multicolumn{3}{|c|}{ Group 5} & \multicolumn{3}{|c|}{ Group 6} \\
\hline & & $n$ & $I$ & $S$ & $n$ & $I$ & $S$ & $n$ & I & $S$ & $n$ & 1 & $S$ & $n$ & 1 & $S$ & $n$ & 1 & $S$ \\
\hline \multirow[t]{2}{*}{1} & U.S. & 310 & 3.696 & -0.014 & & & & & & & & & & & & & & & \\
\hline & Ch. & & 3.529 & 0.027 & & & & & & & & & & & & & & & \\
\hline \multirow[t]{2}{*}{2} & U.S. & 187 & 3.795 & -0.003 & 123 & 3.523 & -0.017 & & & & & & & & & & & & \\
\hline & Ch. & & 3.183 & 0.008 & & 4.122 & 0.015 & & & & & & & & & & & & \\
\hline \multirow[t]{2}{*}{3} & U.S. & 179 & 3.771 & -0.007 & 62 & 4.153 & -0.044 & 72 & 2.89 & $0.093 *$ & & & & & & & & & \\
\hline & Ch. & & 3.156 & 0.003 & & 4.02 & -0.017 & & 4.17 & 0.024 & & & & & & & & & \\
\hline \multirow[t]{2}{*}{4} & U.S. & 116 & 3.407 & -0.007 & 72 & 4.171 & -0.044 & 58 & 2.85 & $0.114^{*}$ & 64 & 4.126 & 0.039 & & & & & & \\
\hline & Ch. & & 3.302 & 0.01 & & 3.997 & -0.022 & & 4.26 & 0.026 & & 3.02 & -0.034 & & & & & & \\
\hline \multirow[t]{2}{*}{5} & U.S. & 95 & 3.308 & 0.016 & 75 & 4.012 & -0.04 & 56 & 2.85 & $0.113^{*}$ & 68 & 4.119 & 0.039 & 16 & 4.519 & -0.086 & & & \\
\hline & Ch. & & 3.267 & 0.008 & & 3.728 & 0.02 & & 4.27 & 0.022 & & 3.002 & -0.037 & & 4.471 & -0.076 & & & \\
\hline \multirow[t]{2}{*}{6} & U.S. & 93 & 3.258 & 0.029 & 69 & 3.997 & -0.032 & 56 & 2.84 & $0.118^{*}$ & 64 & 4.2 & -0.056 & 13 & 4.568 & -0.097 & 15 & 3.894 & $0.187^{*}$ \\
\hline & Ch. & & 3.272 & 0.008 & & 3.801 & 0.018 & & 4.28 & 0.022 & & 3.118 & 0.015 & & 4.532 & -0.087 & & 2.715 & $-0.085^{*}$ \\
\hline
\end{tabular}

Note. Continuously shaded and continuously white columns indicate stable groups across solutions (i.e., groups remained stable as of Model 4$) . * p<.05$ 
Table 4

Values Groups Predicting Positive Youth Development Outcomes

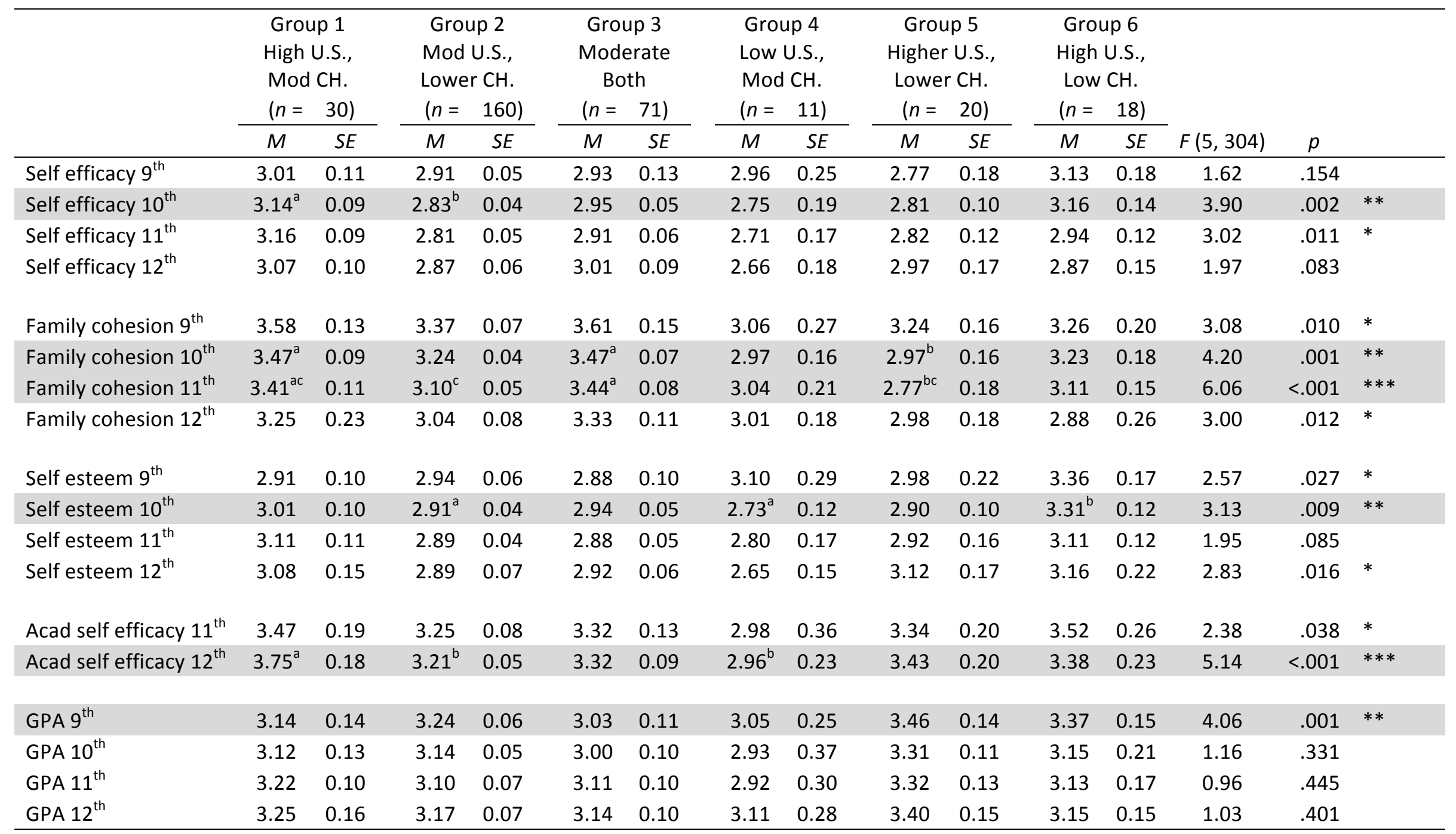

Note. Omnibus differences that reached the adjusted significance level (.01) are highlighted. Entries with different superscripts within a row were significantly different-no superscripts indicates no difference from any other group. ${ }^{*} p<.05, * * p<.01, * * * p<.001$ 
Table 5

Behaviors Groups Predicting Positive Youth Development Outcomes

\begin{tabular}{|c|c|c|c|c|c|c|c|c|c|c|c|c|c|c|c|}
\hline & \multicolumn{2}{|c|}{ Group 1} & \multicolumn{2}{|c|}{ Group 2} & \multicolumn{2}{|c|}{ Group 3} & \multicolumn{2}{|c|}{ Group 4} & \multicolumn{2}{|c|}{ Group 5} & \multicolumn{2}{|c|}{ Group 6} & \multirow[b]{3}{*}{$F(5,304)$} & \multirow[b]{3}{*}{$p$} & \\
\hline & \multirow{2}{*}{$\frac{(n=}{M}$} & 69) & \multicolumn{2}{|c|}{$(n=56)$} & \multicolumn{2}{|c|}{$(n=93)$} & \multicolumn{2}{|c|}{$(n=64)$} & \multicolumn{2}{|c|}{$(n=13)$} & \multicolumn{2}{|c|}{$(n=15)$} & & & \\
\hline & & $S E$ & $M$ & $S E$ & $M$ & $S E$ & $M$ & $S E$ & $M$ & $S E$ & $M$ & $S E$ & & & \\
\hline Self efficacy $9^{\text {th }}$ & 3.03 & 0.09 & 2.93 & 0.12 & 2.83 & 0.06 & 2.96 & 0.08 & 3.26 & 0.24 & 2.64 & 0.14 & 3.37 & .006 & $* *$ \\
\hline Self efficacy $10^{\text {th }}$ & 2.93 & 0.06 & 2.94 & 0.06 & 2.78 & 0.05 & 2.99 & 0.06 & 3.10 & 0.18 & 2.82 & 0.14 & 2.35 & .041 & $*$ \\
\hline Self efficacy $11^{\text {th }}$ & 2.93 & 0.08 & 2.85 & 0.07 & 2.80 & 0.06 & 2.91 & 0.08 & 3.08 & 0.10 & 2.78 & 0.09 & 1.40 & .226 & \\
\hline Self efficacy $12^{\text {th }}$ & 2.94 & 0.07 & 3.04 & 0.11 & 2.89 & 0.08 & 2.85 & 0.08 & 3.05 & 0.20 & 2.75 & 0.12 & 1.68 & .139 & \\
\hline Family cohesion $9^{\text {th }}$ & 3.52 & 0.11 & 3.58 & 0.13 & 3.31 & 0.12 & 3.30 & 0.08 & $4.00^{\mathrm{a}}$ & 0.21 & $2.99^{b}$ & 0.20 & 5.76 & $<.001$ & $* * *$ \\
\hline Family cohesion $10^{\text {th }}$ & 3.37 & 0.08 & 3.44 & 0.08 & 3.23 & 0.06 & 3.16 & 0.07 & $3.63^{\mathrm{a}}$ & 0.13 & $2.98^{\mathrm{b}}$ & 0.16 & 3.59 & .004 & $* *$ \\
\hline Family cohesion $11^{\text {th }}$ & $3.25^{\mathrm{cd}}$ & 0.08 & $3.36^{d}$ & 0.08 & $3.09^{c d}$ & 0.07 & $3.05^{\mathrm{cd}}$ & 0.08 & $3.84^{\mathrm{ad}}$ & 0.20 & $2.81^{b c}$ & 0.17 & 6.28 & $<.001$ & $* * *$ \\
\hline Family cohesion $12^{\text {th }}$ & 3.18 & 0.14 & 3.25 & 0.13 & 3.10 & 0.08 & 2.94 & 0.13 & 3.55 & 0.27 & 2.76 & 0.23 & 3.52 & .004 & $* *$ \\
\hline Self esteem $9^{\text {th }}$ & 2.96 & 0.10 & 2.73 & 0.17 & 2.97 & 0.08 & 3.11 & 0.10 & 3.03 & 0.20 & 3.04 & 0.17 & 3.18 & .008 & * \\
\hline Self esteem $10^{\text {th }}$ & 3.02 & 0.05 & 2.83 & 0.06 & 2.86 & 0.05 & 3.03 & 0.07 & 3.16 & 0.15 & 3.04 & 0.13 & 2.96 & .013 & \\
\hline Self esteem $11^{\text {th }}$ & 2.99 & 0.06 & 2.80 & 0.06 & 2.88 & 0.06 & 2.93 & 0.07 & 3.04 & 0.15 & 3.08 & 0.15 & 1.70 & .135 & \\
\hline Self esteem $12^{\text {th }}$ & 2.95 & 0.09 & 2.83 & 0.07 & 2.95 & 0.07 & 2.97 & 0.13 & 2.82 & 0.20 & 3.18 & 0.16 & 1.61 & .157 & \\
\hline Acad self efficacy $11^{\text {th }}$ & 3.39 & 0.12 & 3.19 & 0.15 & 3.23 & 0.11 & 3.28 & 0.09 & 3.58 & 0.30 & 3.54 & 0.21 & 2.09 & .668 & \\
\hline Acad self efficacy $12^{\text {th }}$ & 3.36 & 0.09 & 3.23 & 0.12 & 3.22 & 0.09 & 3.37 & 0.14 & 3.45 & 0.22 & 3.47 & 0.21 & 1.66 & .143 & \\
\hline GPA $9^{\text {th }}$ & 3.17 & 0.07 & 3.04 & 0.09 & 3.22 & 0.07 & 3.25 & 0.08 & 3.34 & 0.13 & 3.33 & 0.16 & 1.80 & .112 & \\
\hline GPA $10^{\text {th }}$ & 3.20 & 0.07 & 2.86 & 0.12 & 3.12 & 0.08 & 3.13 & 0.08 & 3.24 & 0.20 & 3.35 & 0.18 & 2.36 & .040 & $*$ \\
\hline GPA $11^{\text {th }}$ & 3.24 & 0.07 & 3.00 & 0.14 & 3.12 & 0.09 & 3.06 & 0.09 & 3.26 & 0.17 & 3.18 & 0.17 & 1.25 & .284 & \\
\hline GPA $12^{\text {th }}$ & 3.23 & 0.09 & 3.04 & 0.11 & 3.22 & 0.07 & 3.16 & 0.12 & 3.19 & 0.22 & 3.38 & 0.18 & 1.30 & .264 & \\
\hline
\end{tabular}

Note. Omnibus differences that reached the adjusted significance level (.01) are highlighted. Entries with different superscripts within a row were significantly different - no superscripts indicates no difference from any other group. ${ }^{*} p<.05,{ }^{* *} p<.01,{ }^{* * *} p<.001$ 


\section{U.S. "....... Chinese}
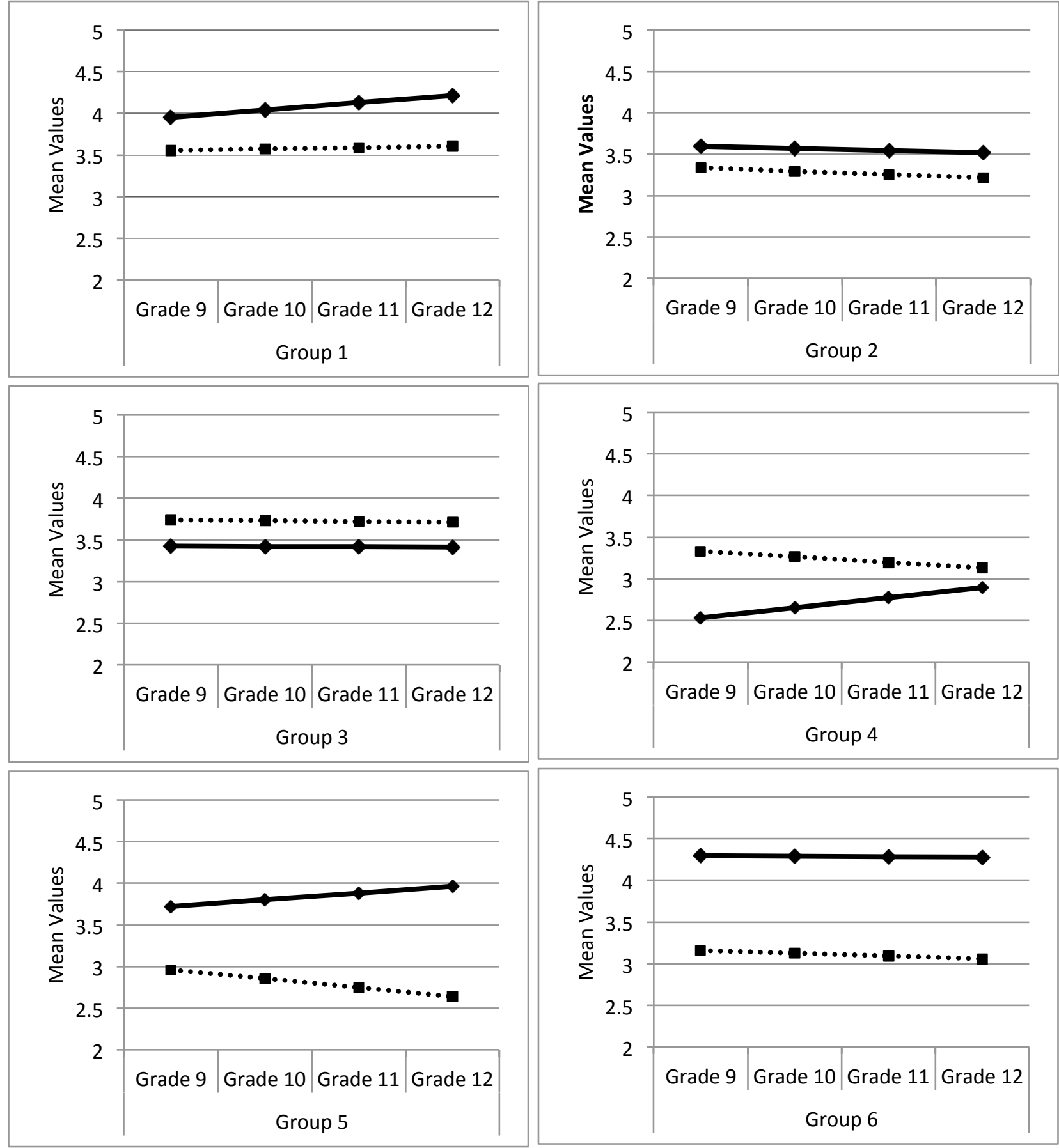

Figure 1. Six-group solution for acculturation values. 


\section{U.S.}

Chinese

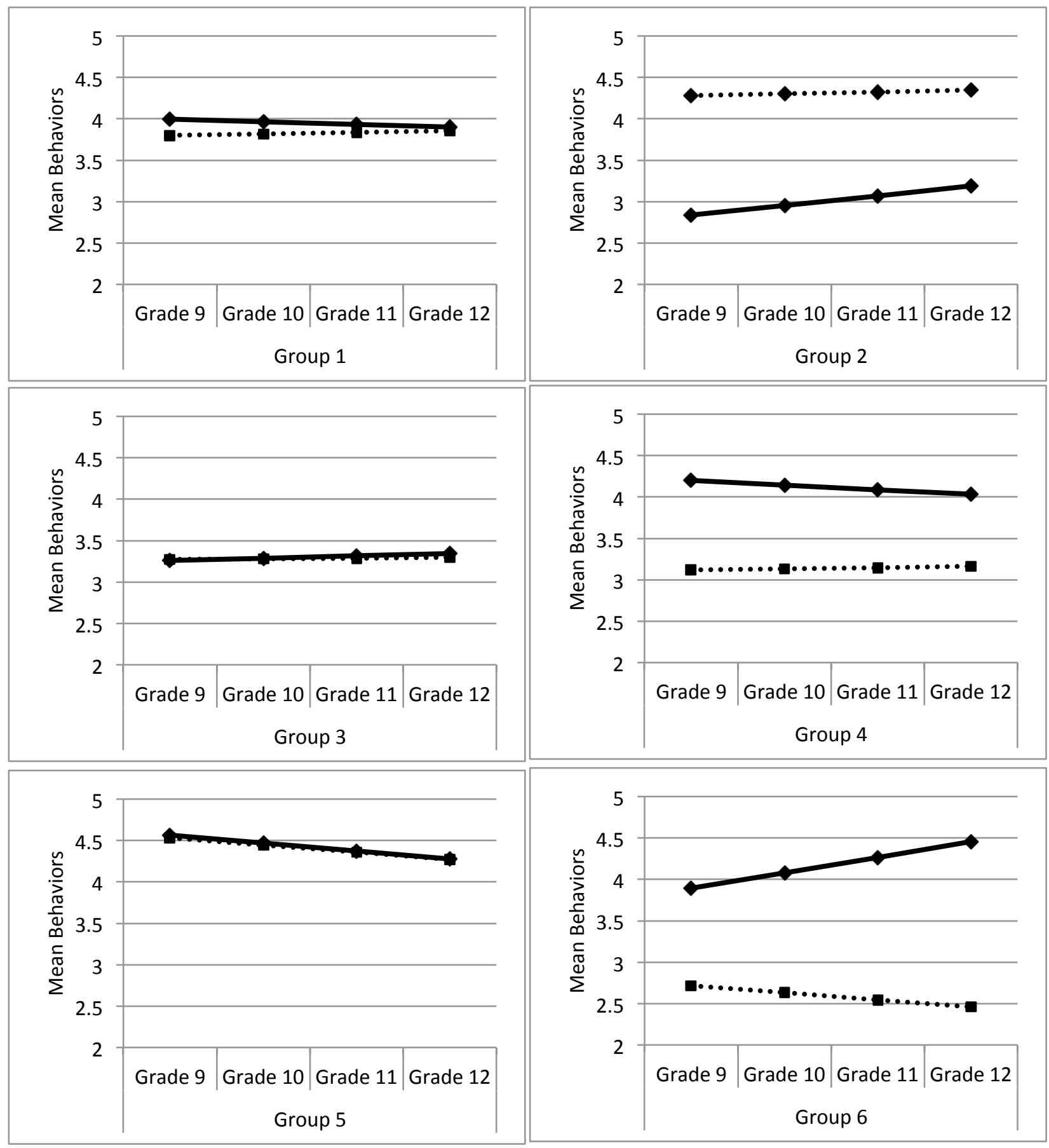

Figure 2. Six-group solution for acculturation behaviors. 